\title{
DEHYDRATION OF 1-OCTANOL TO DI-N-OCTYL ETHER IN LIQUID PHASE WITH SIMULTANEOUS WATER REMOVAL OVER ION EXCHANGE RESINS: EFFECT OF WORKING-STATE MORPHOLOGIES
}

\author{
Roger Bringué, Carles Fité, Montserrat Iborra, Javier Tejero, Fidel Cunill* \\ Chemical Engineering Department. Faculty of Chemistry. University of Barcelona. \\ C/ Martí i Franqués 1, 08028-Barcelona. \\ fcunill@ub.edu
}

\begin{abstract}
The influence of the concentration of polar reactants and products on the working-state morphology of ion exchange catalysts has been investigated over different acidic ionexchange resins for di-n-octyl ether (DNOE) synthesis from 1-octanol dehydration at 423-448 K and atmospheric pressure in a batch reactor equipped with a Dean \& Stark device. By removing water formed 1-octanol conversion was practically complete; the olefin formation being the main secondary reaction. When 1-octanol is completely consumed the working-state morphology of ion exchange resins changes, which influences the selectivities towards products. At this point, for microporous resins all reactions stop while with macroreticular ones DNOE decomposes and significant amounts of olefin dimers appears. The best selectivity to DNOE was found in gel-type and macroreticular resins with low crosslinking degree. Macroreticular resins with medium or high crosslinking give good results in olefin formation.
\end{abstract}

Keywords: 1-octanol, di-n-octyl ether, diesel fuel, alcohol dehydration, ion exchange resins 


\section{Introduction}

Currently European oil industry is making a big effort to adapt the production facilities to maximize the gasoil production and, at the same time, improve its quality, as a consequence of the following facts: the excess gasoline-production capacity; the severe specifications ruled by the 2003/17/EC directive involving cetane number, distillation curve, viscosity, density, cold fuel properties and sulphur content; and the introduction of bio-compounds in the fuel composition ruled by 2009/28/EC and 2009/30/EC directives. An option to upgrade the quality of diesel fuel and fulfil such requirements is the reformulation of gasoil composition by adding suitable oxygenated compounds. Reformulated fuel diesels are expected to have a higher cetane number, lower density, and a lower content of aromatics, PAH (polycyclic aromatic hydrocarbons) and sulphur contents ${ }^{1}$.

It is a well-known fact that blending linear symmetric ethers containing more than nine carbon atoms to commercial gasoil upgrades the quality of the diesel blend ${ }^{2}$. As shown in Table 1, thanks to their high cetane number and fluid properties, DNOE is a good candidate for enhancing blending cetane numbers resulting in a cleaner combustion of diesel fuel and, consequently, in a substantial reduction of pollutants emissions.

In addition to those properties highlighted, blends of ethers and diesel fuels were evaluated from the perspective of fuel performance, stability and material compatibility $^{3,4}$. Finally, it is to be noted that due to the dilution effect, the sulphur and aromatic polycyclic compounds content decreases.

Table 1. Properties of DNOE as diesel fuel

Diesel fuel DNOE

\begin{tabular}{lcc}
\hline Density at $288-293{ }^{\circ} \mathrm{C}\left(\mathrm{kg} / \mathrm{m}^{3}\right)$ & 850 & 806 \\
Boiling point (K) & $443-653$ & 559 \\
Viscosity (cSt) & $3-4$ & - \\
Cetane number ${ }^{*}(-)$ & $48-51$ & 119 \\
Cloud Point $^{*}(\mathrm{~K})$ & $271-278$ & 256 \\
Cold Filter Plugging Point $^{*}(\mathrm{~K})$ & $269-276$ & 258
\end{tabular}


* Blending values.

The main pathway of obtaining linear ethers such as DNOE is the dehydration of the corresponding linear alcohol, 1-octanol in this case. There are several synthesis pathways for obtaining such linear alcohol. One of this is the oxo process by hydroformilation of linear olefins (1-heptene) from fluid catalytic cracking. Another way of preparing 1octanol industrially is by ethylene oligomerization using triethylaluminium followed by oxidation of the alkylaluminium products (Alfol-process). Both petrochemical process might be an option to upgrade refinery streams ${ }^{5}$. Low temperature Fisher-Tropsch syncrude from gas-to-liquid facilities can be also a source of 1 -octanol ${ }^{6}$. On the other hand, 1-octanol is a byproduct of ethanol oligomerization on hydroxyapatite ${ }^{7}$. If used ethanol was produced via biomass fermentation, DNOE could acquire the category of bioether and could be considered as a renewable energy source. Promising works involving 1-octanol synthesis are being developed which uses the biomass-derived platform molecule furfural and acetone ${ }^{8}$ and microbial production of 1-octanol from glucose ${ }^{9}$.

Intermolecular dehydration of primary alcohols to give linear ethers takes place easily in presence of acid catalysts ${ }^{10}$. Considering the advantages of using acid solid catalysts, some research work on DNOE was conducted in liquid phase over ion exchange resins catalysts without formed water removal ${ }^{11-13}$. Over zeolite beta, 1-octanol dehydrates selectively to octenes ${ }^{14}$. Zeolita Beta and Nafion-H have been also tested in liquid phase by using batch reactor equipped with a Dean \& Stark device for formed water removal. On Nafion-H, 1-octanol conversion at $418-423 \mathrm{~K}$ was $97 \%$ and selectivity to DNOE was $100 \%{ }^{15}$. Despite this excellent result, the industrial use of Nafion-H is economically questioned, because its price might be more than one order of magnitude respect to sulfonated resins at lab scale.. Increasing interest in biomass valorization has also triggered a research for new catalytic routes to transform biomass-based derivatives in octyl-ethers to be used as transportation fuels. Thus, two catalytic routes has been recently proposed for the synthesis of bio-based long alkyl chain ethers: the telomerization of 1,3butadiene with biomass-based glycols affords linear octyl ethers with high yields (up to $80 \%$ ) using a homogeneous Pd-based catalyst; and the direct etherification with long linear alkenes with $\mathrm{H}$-Beta zeolites ${ }^{16}$

As aforementioned ${ }^{11-13}$, ion exchange resins could be a suitable catalyst for the synthesis of di-n-octyl ether. However, in the range $413-453 \mathrm{~K}$ it takes tens of hours to get the 
equilibrium conversion (higher than 96\%) with selectivities to DNOE higher also than 95\% for some resins. By-products found were octenes and very small amounts of branched ethers. It would be necessary a temperature rise to increase the reaction rate to an appropriate level for industrial purposes. Unfortunately, literature quoted above shows that a suitable temperature for higher linear alcohols dehydration reaction $(>423 \mathrm{~K})$ to linear ethers is greater than the maximum operational temperature for standard acidic resins. Another drawback to be considered is that etherification on acidic ion exchange resins is inhibited by water, which is a reaction product from the alcohol etherification reaction and from alcohol dehydration to olefins ${ }^{17}$.

One aim of the present work is to find the experimental conditions and the catalyst that allow to increase the ether production by dehydration of higher linear alcohols catalyzed by acidic ion exchange resins. Continuous water removal would shift equilibrium conversion to total conversion. Experimental work will be performed in a bath reactor equipped with a Dean \& Stark device for water removal.

\section{Experimental Section}

\subsection{Chemicals.}

1-octanol (99.5\% pure, GC), 1-(octyloxy)octane or DNOE (99\% pure, GC) and 1-octene and 2-octene ( $>97 \%$ pure, GC) were supplied by Across Organics, Sigma Aldrich and Fluka, respectively

\subsection{Catalysts.}

As catalysts gel and macroreticular acidic resins will be used to check their performance in the circumstances above mentioned for 1-octanol dehydration. The tested catalysts were acidic polystyrene-divinylbenzene (PS-DVB) based resins: Amberlyst 15 (A-15), Amberlyst 16 (A-16), Amberlyst 35 (A-35), Amberlyst 36 (A-36), Amberlyst 39 (A-39), Amberlyst 46 (A-46) and Amberlyst 70 (A-70) from Rohm and Haas; Purolite CT-175, CT-224, CT-252, CT-276 from Purolite; and Dowex 50x4 (DOW50Wx4) 200-400 mesh from Aldrich. They have been chosen to cover a wide range of macroreticular and geltype resins properties and characteristics. The resins were delivered in wet form. They were activated at $383 \mathrm{~K}$, first at atmospheric pressure and then overnight under vacuum at the same temperature. Final water content after drying was less 3\% w/w (Karl-Fisher titration). Commercial particles sizes were use in all the experiments. 


\subsection{Apparatus}

The experiments were carried out in a $1 \mathrm{~L}$ glass batch reactor at atmospheric pressure. One of the openings was connected to a reflux consisting of a Dean \& Stark burette to separate the produced water and a condenser cooled by water. Another opening was used to fit the temperature probe. The reactor was heated to boiling point of the mixture and stirred using a heat-stir supplied by Stuart (model SB162).

\subsection{Analysis}

Liquid samples, taken from the of reactor after finishing experiment, were analysed in a gas chromatograph (Hewlet-Packard GC 6890A) equipped with a TCD detector, that allows to measure the water content, using a $50 \mathrm{~m} \mathrm{x} 0.2 \mathrm{~mm}$ x $0.5 \mu \mathrm{m}$ methyl silicone HP 90915-001 capillary column. It was temperature programmed with 6 min initial hold at $45^{\circ} \mathrm{C}$ followed by a $30^{\circ} \mathrm{C} \mathrm{min}^{-1}$ ramp up to $180^{\circ} \mathrm{C}$ and held for $5 \mathrm{~min}$. Helium was used as carrier gas at a total flow rate of $30 \mathrm{~mL} \mathrm{~min}^{-1}$.

\subsection{Procedure and calculations}

$400 \mathrm{~g}$ of 1-octanol and $9 \mathrm{~g}$ of dry catalyst were charged into the reactor, stirred ( $700 \mathrm{rpm}$ ) and heated to the desired temperature. Initial time of experiment was chosen when the reacting mixture began to boil. Vapours were cooled and condensed in a Dean \& Stark burette. Water, 1-octanol and olefins were collected and the organic phase (mainly 1octanol) was returned to the reactor. The experiment finished when the separated water amount equalled that estimated by stoichiometry for full conversion of 1-octanol. At the end of experiment the remaining liquid in the reactor and the burette content (aqueous and organic phase) were analysed.

For each experiment, conversion of 1-octanol, selectivity and fractional yield to DNOE were calculated. The 1-octanol conversion is defined as:

$$
\mathrm{X}_{1-\mathrm{octanol}}=\frac{\text { reacted mole of } 1 \text {-octanol }}{\text { initial mole of } 1 \text {-octanol }}
$$

Selectivity of 1-octanol forward product $\mathrm{j}$ (DNOE, $\mathrm{C}_{8}$ olefins, and branched ethers) is defined as: 


$$
\mathrm{S}_{\mathrm{j}}=\frac{\text { reacted mole of 1-octanol to form } \mathrm{j}}{\text { reacted mole of 1-octanol }}
$$

Selectivity towards alkenes (1-octene, 2-octene, etc., originated by intramolecular dehydration of 1-octanol) and branched ethers other than DNOE (1,2 oxybis octane, 1,3 oxybis octane, etc., originated by alcohol-olefin reactions) was defined analogously.

Fractional yield to DNOE is defined as:

$$
\mathrm{Y}_{\mathrm{DNOE}}=\mathrm{X}_{1-\mathrm{octanol}} \cdot \mathrm{S}_{\mathrm{DNOE}}=\frac{\text { reacted mole of 1-octanol to form DNOE }}{\text { initial mole of 1-octanol }}
$$

In all experiments, mass balance was accomplished within $2 \%$. It was assumed that experimental results could be affected by thermal deactivation of resins, but since the experiment duration was $7 \mathrm{~h}$, the deactivation can be considered negligible.

\section{Results and discussion}

At those analitical conditions afore mentioned the chemicals detected and their retention times can be observed in Table 2.

Table 2. Chemical detected in the reacting system and their retention times

\begin{tabular}{|c|c|}
\hline Chemical & Retention time (min.) \\
\hline Water & 4.55 \\
1-octene & 8.52 \\
4-octene & 8.59 \\
3-octene (trans) & 8.64 \\
3-octene (cis) & 8.69 \\
2-octene (trans) & 8.74 \\
2-octene (cis) & 8.90 \\
1-octanol & 13.03 \\
Ether 1 & 20.05 \\
Ether 2 & 20.24 \\
Ether 3 & 20.41 \\
Di n-octil ether & 21.32 \\
\hline
\end{tabular}

As alcohols different of 1 -octanol were not detected, branched ethers $(1,2,3)$ formed came from the reaction between 1-octanol and olefins formed in the system. As we do not have 
standards for all octenes they were lumped as 1-octene. The obtained distribution o products and byproducts suggest the reaction scheme in Figure 1.

\section{FIGURE 1}

Following is a summary of the most important points of the features and behaviour of acid resins as catalysts. In this way, it is intended to facilitate the discussion of results. It is expected a very different performance of these types of resins due to the difference in the morphology of their polymer skeletons because the swelling of resins in contact with reaction environment. Swelling is a key factor for the accessibility to the catalytic centers. This is true also for macroreticular or macroporous resins having a porosity independent of swelling of the polymer matrix. However, only a little fraction of acid centers is located on the walls of such permanent porous. Macroreticular resins are polymers obtained by copolymerization of styrene and divinylbenzene (PS-DVB) in presence of a solvent called "porogen". When the solvent is taken out of polymer permanent pores appear. On the contrary, in absence of a solvent, microporous or gel-type resins are obtained with no permanent pores in dry state. Then, macroreticular resins consist of large agglomerates of gel microspheres, which, in turn, show smaller nodules that are more or less fused together ${ }^{18}$. Figure 2 shows a picture of the morphology of both kinds of resins in dry and swollen state. In swollen state gel-type resins contain two groups of pores: micropores for inaccesible part of matrix and new mesoporous which desappear on deswelling. Macroreticular resins shows three types of pores: micropores of non swelling inaccesible part of polymer matrix, new mesoporous and macroporous coming from permanent porosity.

A useful description of morphology on swollen state can be obtained from Inverse Steric Exclusion Chromatography (ISEC) ${ }^{19}$. Those macroporous, so-called "true pores", and mesoporous are interpreted in terms of the model of cylindrical pores. System of smaller pores is described in terms of the model proposed by Ogston ${ }^{20}$ in which micropores are seen as spaces between randomly oriented rigid rods representing the polymer chains. The specific volume of swollen polymer (volume of the free space plus that occupied by the skeleton, $\mathrm{V}_{\mathrm{sp}}$ ) is the characteristic parameter of such description. The Ogston model distinguish polymer zones of different density or polymer chain concentration in terms of total rod length per volume unit of swollen polymer, $\mathrm{nm} / \mathrm{nm}^{3}$. 
Table 3 shows $V_{\text {sp }}$ of resins to be tested. It can be seen that $V_{\text {sp }}$ decreases as DVB\% increases both for gel-type and macroreticular resins. It is worth noticing that low $\mathrm{V}_{\mathrm{sp}}$ values are indicative of high density of polymer mass in swollen state and as result small and stiff spaces are available in the gel phase equivalent to pores lesser than $1 \mathrm{~nm}$, poorly accessible spaces even for small molecules. Conversely, high $\mathrm{V}_{\text {sp }}$ values are associated with low density of polymer mass, and imply relatively large spaces equivalent to pores up to $8 \mathrm{~nm}$.

\section{FIGURE 2}

Main properties and structural parameters of resins in dry state and swollen in water are shown in Table 3 and Figures 3 and 4. Properties in dry state can be useful to approximate non-polar reacting medium, while for a polar one properties are better described by those obtained in swollen state. Approaches based on porosimetric information obtained by conventional methods (nitrogen adsorption/desorption, mercury porosimetry) are appropriate to explain what is happening in non-polar media, but they are unable to explain the situation in presence of swelling solvents. As depicted before on swelling the morphology of resins changes and new non-permanents porous appear.

As shown in Table 3, macroreticular resins with high and medium crosslinking degree have permanent macropores in dry state. It is to be noted that Amberlyst 16, 39 and 70 resins with DVB\% close to 8 show a very low BET surface area. However, comparing with ISEC data, it is clear that those new pores are open in aqueous media with surface area ranging between 132 and $194 \mathrm{~m}^{2} / \mathrm{g}$, and pore volume between 0.36 and $0.91 \mathrm{~cm}^{3} / \mathrm{g}$. Both parameters are far higher than those for dry state showing that new intermediate pores (mesopores) are accessible. Correspondingly, pore diameter in aqueous medium is lower than in dry state. Fig. 3 and 4 show the pore distribution in the swollen gel phase, quite representative of the morphology of swollen acidic resins in aqueous solution. $\mathrm{V}_{\mathrm{sp}}$ decreases as DVB\% increases both in gel-type and macroporous resins. It is seen that swollen gel phase of microporous and low-crosslinked macroreticular resins show chains concentrations of $0.4-0.8 \mathrm{~nm}^{-2}$, typical of low dense polymer mass; whereas medium and high crosslinked macroreticular resins show densities of 1.5 and $2 \mathrm{~nm}^{-2}$, respectively, typical of high dense polymer mass.

The real morphology depends on the reaction environment. It will be closer to dry state when the resins are collapsed, which can occur in non-polar medium, while it will be closer to swollen state when the resins are in efficient swelling solvents. On the other 
hand, as enthalpic effects can be present due to interactions between reactants and chains of the polymer matrix, the knowledge and a quantitative description of the actual swollenstate of resins is very difficult, if not impossible.

\section{FIGURE 3}

\section{FIGURE 4}

The reaction mixture for 1-octanol dehydration contains at the beginning only alcohol and therefore, a polar reaction environment with a reasonably swelling. At the end, two extreme options of composition are viable depending on the resins behaviour. The first could contain a very small amount of water (the majority was eliminated by the Dean $\&$ Stark system) and a majority of olefins, and consequentlty the reaction environment would be non-polar and the resins would be rather collapsed. The second option could also contain a small amount of water and ethers, that to say, a polar environment but much lesser than for the alcohol. This way, changes of the proportions of the swelling and nonswelling components could affect the working-state morphology of the resins and as a result different amounts of products and byproducts could be formed. In addition, on decreasing the alcohol concentration products can compete for the sulfonic acid groups, which meant a shift from a slower mechanism of specific acid catalysis (catalysis by protons) to a more efficient mechanism of general acid catalysis (catalysis by cluster of sulfonic groups $)^{21}$.

During catalytic experiments the ion exchange resin catalysts are swollen when water and/or alcohol are present. As the amount of water and alcohol are changing during the experiments the real morphology can not be explained neither by ISEC nor by dry sate. It will be between these extreme situations. However, ISEC could explain the morphology in the first stage of the experiments when the first amount of formed water is not even retired from the media and at the same time the amount of alcohol is high.

Table 3. Characteristics and structural parameters of resins in dry state and swollen in water

\begin{tabular}{|c|c|c|c|c|c|c|c|c|c|}
\hline \multirow[t]{2}{*}{ Type $^{a}$} & \multirow[t]{2}{*}{ Catalyst } & \multirow[t]{2}{*}{ Sulfonation $^{\mathrm{a}}$} & \multirow{2}{*}{$\begin{array}{l}\mathrm{T}_{\max } \\
(\mathrm{K})\end{array}$} & \multirow{2}{*}{$\begin{array}{c}{\left[\mathrm{H}^{+}\right]^{\mathrm{b}}} \\
(\mathrm{mol} / \mathrm{kg})\end{array}$} & \multirow[t]{2}{*}{ DVB $\%$} & \multicolumn{2}{|c|}{ Dry state } & \multicolumn{2}{|c|}{ Swollen in water } \\
\hline & & & & & & $\mathrm{Sg}_{\mathrm{g}}^{\mathrm{d}}$ & $\mathrm{V}_{\mathrm{g}}^{\mathrm{e}}$ & $\mathrm{S}_{\mathrm{g}}$ & $\mathrm{V}_{\mathrm{sp}}\left(\mathrm{cm}^{3} / \mathrm{g}\right)$ \\
\hline & A-46 & SS & 423 & 0.87 & 25 & 57 & 0.26 & 186 & 0.19 \\
\hline$\overline{0} \overline{0}=$ & A-35 & OS & 423 & 5.32 & 20 & 34 & 0.21 & 199 & 0.50 \\
\hline 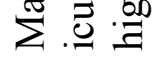 & A-15 & MS & 393 & 4.81 & 20 & 42 & 0.33 & 192 & 0.62 \\
\hline
\end{tabular}




\begin{tabular}{|c|c|c|c|c|c|c|c|c|c|}
\hline & CT-175 & MS & 403 & 4.98 & High & 28 & 0.30 & 157 & 1.00 \\
\hline & CT-276 & OS & 408 & 5.3 & $16-18$ & 23.5 & 0.21 & 176 & 0.77 \\
\hline \multirow{6}{*}{ 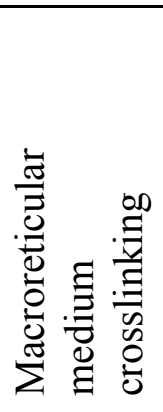 } & CT-252 & OS & 403 & 5.4 & Medium & 22.4 & 0.22 & 132 & 0.98 \\
\hline & A-16 & MS & 393 & 4.80 & 12 & 1.7 & 0.013 & 46 & 1.14 \\
\hline & A-36 & OS & 423 & 5.40 & 12 & 21 & 0.14 & 68 & 1.26 \\
\hline & A-39 & MS & 403 & 4.81 & $7-8$ & 0.09 & $3 \cdot 10^{-4}$ & 56 & 1.64 \\
\hline & A-70 & MS & 463 & 2.65 & $7-8$ & 0.018 & - & 66 & 1.15 \\
\hline & A-70 & MS & 463 & 2.65 & $7-8$ & 30 & 0.15 & - & - \\
\hline \multirow{2}{*}{$\overline{0}$} & CT-224 & OS & 423 & 5.34 & 4 & 0.95 & $1 \cdot 10^{-3}$ & - & 1.86 \\
\hline & DOW50Wx4 & MS & 423 & 4.95 & 4 & 0.011 & - & - & 1.90 \\
\hline
\end{tabular}

${ }^{a}$ Monosulfonated (MS), oversulfonated (OS), and sulfonated only near the polymer surface (SS)

${ }^{\mathrm{b}}$ Acid capacity. Titration against standard base

${ }^{c}$ By adsorption-desorption of $\mathrm{N}_{2}$ at $77 \mathrm{~K}\left(\mathrm{~N}_{2}\right.$ for $\mathrm{S}_{\mathrm{g}} \geq 1 \mathrm{~m}^{2} / \mathrm{g} ; \mathrm{Kr}$ for $\left.\mathrm{S}_{\mathrm{g}}<1 \mathrm{~m}^{2} / \mathrm{g}\right)$

${ }^{\mathrm{d}}$ BET method

e Volume of $\mathrm{N}_{2}$ adsorbed at relative pressure $\mathrm{P} / \mathrm{P}_{0}=0.99$

${ }^{\mathrm{f}}$ ISEC method

g Rinsed with methanol, after with toluene and finally with isooctane

It is noteworthy that swelling in 1-octanol and water are similar. Swelling by water is due to interaction with sulfonic groups, but in the case of higher alcohols an additional interaction of aliphatic part of the alcohol molecule with polymer chains is possible ${ }^{22}$. The experiments were carried out at atmospheric pressure and at the boiling point of the reacting system (423-448K). Fig. 5 shows the reactor temperature evolution versus the amount of removed water and collected in the Dean \& Starch burette. The curve obtained for CT 224 was typical for microporous resins and also for macroporous resins with low DVB content. That of A-36 is typical for macroreticular of medium and high DVB content. Several stages can be observed in that Figure. Initially, temperature was about $433 \mathrm{~K}$ for A-36 and $440 \mathrm{~K}$ for CT-224 while the boiling point of 1-octanol is $468 \mathrm{~K}$. It should be noted that zero time was set when the first drop of condensed vapour appeared. Therefore, at that moment, some extension of main and secondary reaction could have taken place. The presence of low boiling point water (373K) and 1-octene (b.p. 394K) compared to 1-octanol and formed DNOE (b.p. 559K), reduces the boiling point of the reacting mixture. As long as initially formed water is both captured by the resin and withdrawn from the reacting system by the Dean \& Starch burette, the reactor temperature increases due to DNOE accumulation. 


\section{FIGURE 5}

As long as there was alcohol in the reacting system water followed forming, so there was always some little amount of water in the resin. Under these conditions, the resin was swollen and, as a result, many acid centers were accessible. It can be seen that the curve for resin CT-224 goes over that for A-36 showing that more products of higher boiling point namely DNOE are formed. The fact that CT-224 has a less dense gel-phase compared with A-36, according to Fig 3 and 4, allows that big and heavy molecules can be easier formed and diffuse towards the liquid phase. On the other side, A-36 with a denser gel-phase does not permit that DNOE permeates which facilitates olefins formation. This explanation can be confirmed by composition values of Fig.5. This behavior remains throughout the experiment.

When about $10 \mathrm{~mL}$ of water had been removed from the system, temperatures for both reacting systems went through a maximum showing that from this point olefin production was faster than DNOE and the amount of lower boiling points products prevailed, as composition values presented in Fig 5 show. Hence, reactor temperatures decreased.

At about $40 \mathrm{~mL}$ of removed water, the temperature profiles reached their minimum value: about $437 \mathrm{~K}$ for the system catalyzed by CT-224, and $423 \mathrm{~K}$ for A-36. At this point it is when we find the major differences among catalysts performance: over microporous and macroreticular with low DVB content ion-exchange resins (represented by CT-224) all reactions stopped and no more water was produced, whereas macroreticular with medium and high DVB content ion-exchange resins (represented by A-36) the production of water continued. Considering the stochiometry of the reactions ( 1 mol of water formed per 1 mol olefin/ether) when all alcohol reacts about $37.5 \mathrm{~mL}$ of water should be formed. This values is in good agreement with the amount of about $40 \mathrm{~mL}$ observed in Fig. 5 collected in the Dean\&Stark device. Therefore, the minimum temperature coincided with the complete consumption of 1-octanol. The different behaviour of the resins could be explained by their morphology. CT-224 has no permanent pores in the dry state, so when all the alcohol and water was consumed, and removed respectively, its structure collapses, what hinders the accesibility to active sites in gel-phase, and then the reaction stops.

FIGURE 6 
On the contrary, what happened with A-36 after the minimum of temperature (or when $40 \mathrm{~mL}$ of water was removed) was completely different: the system continued reacting and the temperature rised again. As noted in Fig. 5, obtained DNOE decreased a lot in that section, from 55.7 to $13.8 \%$, the amount of dimers increased significantly whereas those of olefins and residual 1-octanol decreased slightly. This sharp change of the beaviour of the two resins, CT-224 and A-36, is an immediate consequence of their different morphlogy. As said, CT-224 collapsed, but for A-36 the reduced number of active sites that even can be reached through the permanents pores are enough to decompose DNOE in order to acomplish its chemical equilibrium constant value ${ }^{13}$, which is about 25 at $430 \mathrm{~K}$. DNOE decomposes and results in olefines and alcohol formation. These olefins together with those produced from alcohol dehydration dimerized. As before, water was removed as produced, but some amount remained to swell the resins. Again the composition backs up the obtained results since the amount of water collected was about $55 \mathrm{~mL}$ (Fig. 6) and that calculated form the stoichiometry was a bit higher than $50 \mathrm{~mL}$. Details about octenes oligomerization catalyzed by ion exchange resins and why dimers are the main oligomer can be found elsewhere ${ }^{23,24}$.

Figure 7 shows the evolution of the temperature versus the amount of water collected for all tested resins. In general, the same behaviour between the two big families of ion exchange resins was observed. Gel-type resins trended to produce less olefins and consequently less amount of water and the production of water stopped when all the alcohol was consumed. On the other hand, macroreticular resins produced more olefins and less ethers and, hence, more water. Fig. 7 shows these general trends and also differences, even within the same family of resins due to their different morphology, particularly gel density. Looking carefully at this figure, catalysts can be subdivided into three main groups. The first one, which presents the lower temperature profiles, is formed by the macroreticular resins A-15, A-35. CT-175 and CT-276, all of them with high content of DVB $\%$, low volume of gel-phase (Table 3) and a very high density of polymer of $1.5 \mathrm{~nm}^{-2}$ (Fig.3). Thus, it is not surprising that this group favoured relatively small molecules formation like olefins compared to DNOE. It should be highlighted that these resins gave a high DNOE selectivity just at the minimum temperature of the profile, when the alcohol was totally consumed, i.e., when about $40 \mathrm{~mL}$ of water was removed from the reactor. The lower DNOE selectivity values for A-35 and CT-175 could be explained by 
a high dense phase combined with low water content, coming from dehydration of octanol originated from DNOE decomposition.

A-16, A-36 and CT-252 form the second group with a similar behaviour to the first one, but with a higher temperature profile corresponding to a higher content of ethers. Their less dense gel phase structure facilitates a higher accessibility to acid sites of big molecules with higher boiling point. The last group is formed by microporous and macroreticular resins of low crosslinking. They behave as CT-224, being the ethers formation the main reaction. As already said for CT-224, reactions do not proceed when all the alcohol is consumed. At this point, it is to be noted that the macroreticular resins of low DVB\% began to show, but in a very bit extension, the tendency to keep on reacting now by decomposition of DNOE and dehydrating the formed alcohol. Less dense swollen resins, with wide spaces between polymer chains, allow permeation of 1-octanol, DNOE outside permeation. Therefore, dehydration to DNOE is favoured on gel-type and macroreticular resins with low DVB\%. Besides, it seems that supplementary $-\mathrm{SO}_{3} \mathrm{H}$ groups in oversulfonated resins contribute to hold the polymer chains closer by the presence of additional hydrogen bonds, which would explain selectivities to DNOE lower than those of conventionally sulfonated resins.

\section{FIGURE 7}

A-46 deserves a special discussion, because it is surface sulfonated only. Its sulfonation is only limited to the surface layer of polymer beads and a full sulfonation is expected for that thin zone. However, as a result, its average acid capacity extended to the bead is rather low, and therefore its catalytic activity. Besides, the gel-phase of the thin layer can also show local medium to high density as can be seen in Fig.4. So, in polar medium practically all the active sites are accessible: there is a lot of surface to access to the gel phase. However, in non-polar medium only part of the actives sites located near the surface of the gel-phase can be accessible. All of these elements mean that results obtained with A-46 are difficult to explain. The presence of very low and medium density (Fig. 4) could explain the DNOE and branched ethers formation preferentially. After 7 hours the 1-octanol conversion was full except for A-46, which gave a value of 59\%. To sum up, while there is alcohol non reacted and captured water by the resins, the amount of produced DNOE decreases when the DVB\% of the resins increases or, in other words, when $V_{\text {sp }}$ decreases. 
Table 4. Selectivities and yields obtained for all resins tested after $7 \mathrm{~h}$ on $9 \mathrm{~g}$ of resin at boiling point and atmospheric pressure

\begin{tabular}{|c|c|c|c|c|c|c|}
\hline Catalyst & $\mathbf{X}_{\text {1-octanol }}(\%)$ & S $_{\text {DNOE }}$ & Solefins & S $_{\text {Dimers }}$ & S $_{\text {B ethers }}$ & $\mathbf{Y}_{\text {DNOE }}$ \\
\hline A-15 & 100 & 44.2 & 38.7 & 17.1 & - & 44.2 \\
\hline A-16 & 100 & 49.4 & 26.3 & 24.3 & - & 49.4 \\
\hline A-35 & 100 & 17.4 & 32.9 & 49.6 & - & 17.4 \\
\hline A-36 & 100 & 13.2 & 39.1 & 47.7 & - & 13.2 \\
\hline A-39 & 100 & 48.1 & 27.4 & 24.5 & - & 47.8 \\
\hline A-46 & 59 & 85.6 & 10.6 & - & 3.8 & 49.6 \\
\hline A-70 & 100 & 53.7 & 30.1 & 16.2 & - & 53.5 \\
\hline CT-175 & 100 & 44.6 & 27.8 & 27.6 & - & 44.6 \\
\hline CT-224 & 100 & 63.1 & 28.3 & 8.6 & - & 62.8 \\
\hline CT-252 & 100 & 45.9 & 25.4 & 28.6 & - & 45.9 \\
\hline CT-276 & 100 & 37.2 & 27.8 & 34.9 & - & 37.1 \\
\hline DOW50Wx4 & 100 & 54.7 & 26.4 & 18.9 & - & 54.6 \\
\hline
\end{tabular}

Comparing similar resins with different degree of sulfonation, e.g., A-15 with A-35 or A16 with A-36 (the former conventionally sulfonated, the latter oversulfonated), we can conclude that oversulfonation does not favour the DNOE formation (Table 4). It seems that oversulfonation increases the stiffness of the matrix and hinders the accessibility towards acid sites.

As a whole, it can be concluded that the best DNOE yield was obtained by gel-type resins with low DVB\%, CT-224 being the best one. Fig. 8 displays the water production throughout the experiment and can be used as a relative measure of the activity of tested resins. It is necessary to bear in mind that water is produced from two parallel reactions with different stoichiometry. Production of 1 mole of water needs 2 moles of 1-octanol in the etherification reaction and 1 mole of 1 -octanol in the olefin production reaction. From the slopes of the curves we can infer that the most active resins, from a water formation point of view, were the macroreticular resins A-35, A-36 and A-39. The two first are oversulfonated, whereas the third is conventional sulfonated, but with a high acid capacity. The less active resin was A-46, which has the low acid capacity of the assayed reins. Thus, it might be concluded that the higher acid capacity the greater water formation rate.

FIGURE 8 
FIGURE 9

Figure 9 plots the selectivity to products versus $\left[\mathrm{H}^{+}\right] / \mathrm{V}_{\text {sp }}$ (acid sites per volume unit of swollen gel phase) for those catalysts that reach complete 1-octanol conversion, i.e. all resins except A-46. As seen, SDNOE correlates well with $\left[\mathrm{H}^{+}\right] / \mathrm{V}_{\text {sp: }}$ : selective resins to DNOE have low $\left[\mathrm{H}^{+}\right] / \mathrm{V}_{\text {sp }}$ values, whereas less selective, namely A-36, A-35, CT-276 and $\mathrm{CT} 175$, show high $\left[\mathrm{H}^{+}\right] / \mathrm{V}_{\text {sp }}$ values. It is to be noted that the selectivity to $\mathrm{C} 8$ olefins did not depend on this parameter, since same level was achieved over all resins. Selectivity to dimers show the opposite trend than selectivity to DNOE: more dimers were produced over the resins with a stiffer structure and high acid capacity, such as A-35. The highest value of DNOE selectivity and the lowest value for olefins selectivity corresponds to A46, wich, as said before, has a special morphology, and did not reach complete conversion.

\section{Conclusions}

The paper shows how important is the polarity of the reacting medium on the behaviour of the ion exchange resins as catalysts. Polarity affects the swelling of the resin and, as a result, it modifies the accesibility to active sites during the reaction progress.

Gel-type resins are the most appropiate to obtain large molecules, like DNOE. In presence of 1-octanol and while water is not totally removed, the resin is swollen and the acid sites into the gel-phase are accesible. When the alcohol is consumed and water removed the resins collapsed and the reactions stop. In macroreticular resins, its permanent structure of pores allows the reactions to continue after the alcohol is consumed. Thus, if the goal of 1-octanol dehydration is the production of DNOE, microporous resins are preferred, whereas, if the goal is to obtain olefins and diolefins, the candidate resins are oversulfonated macroreticular of medium to high crosslinking content. .

\section{Acknowledgements}

The authors thank Dow Chemical (Rohm and Haas, France) and Purolite for providing ion exchange resins samples. In addition, it should be noted that this research did not 
receive any specific grant from funding agencies in the public, commercial, or non-forprofit sectors

\section{Nomenclature}

$\mathrm{S}_{\mathrm{B} \text { ethers }}$ selectivity to branched ethers (\%)

SDimers selectivity to dimers (\%)

SDNOE selectivity to DNHE (\%)

Solefins selectivity to olefins (\%)

$\mathrm{S}_{\mathrm{g}} \quad$ ISEC surface area $\left(\mathrm{m}^{2} / \mathrm{g}\right)$

$\mathrm{S}_{\mathrm{g}}^{\mathrm{e}} \quad$ BET surface area $\left(\mathrm{m}^{2} / \mathrm{g}\right)$

$\mathrm{t}$ time $(\mathrm{h})$

$\mathrm{T}_{\max }$ maximum temperature operation

$\mathrm{V}_{\mathrm{g}}^{\mathrm{f}} \quad$ Absortion of $\mathrm{N}_{2}$, pore volume $\left(\mathrm{cm}^{3} / \mathrm{g}\right)$

$\mathrm{Vg}_{\mathrm{g}} \quad$ ISEC pore volume $\left(\mathrm{cm}^{3} / \mathrm{g}\right)$

$\mathrm{V}_{\mathrm{sp}} \quad$ specific volume of the swollen polymer phase $\left(\mathrm{cm}^{3} / \mathrm{g}\right)$

$\mathrm{Y}_{\mathrm{DNOE}} \quad$ fractional yield to DNOE (\%)

Acronyms

DNOE di-n-octyl ether

\section{References}

1. H.G. Pöttering, P. Necas, Directive 2009/30/EC, Off. J. Eur. Union, 140 (2009) 88112.

2. G.C. Pecci, M.G. Clerici, F. Giavazzi, F. Ancillotti, M. Marchionna, R. Patrini, Oxygenated Diesel Fuels. Part 1- Structure and Properties Correlation, IX Int. Symp. Alcohol Fuels, 1 (1991) 321-326.

3. F. Giavazzi, D. Terna, R. Patrini, F. Ancillotti, G.C. Pecci, R. Trerè, M. Benelli, "Oxygenated Diesel fuels. Part 2- Practical aspects of their use", IX Inter. Symp. Alcohol Fuels, 1 (1991) 327-335. 
4. J. Van Heerden, J.J. Botha, P.N.J. Roets,"Improvement of diesel performance with the addition of linear ethers to diesel fuels", 12th Int. Symp. Alcohol Fuels, 1, 188-199 (1998).

5. J. Falbe, H. Bahrmann, W. Lipps, D. Mayer (2011), Ullmann's Encyclopedia of Industrial Chemistry, Weinheim: Wiley-VCH, $7^{\text {th }}$ ed., vol. 2, p. 235.

6. R.J.J. Nel, A. de Klerk, Ind. Eng. Chem. Res., 48 (2009) 5230-5238.

7. T. Tsuchida, S. Sakuma, T. Takeguchi, W. Ueda, Ind. Eng. Chem. Res., 46 (2006) $8634-8642$

8. J. Julis, W. Letner, Angew. Chem. Int. Ed., 51 (2012) 8615-8619.

9. M.K. Akhtar, H. Dandapani, K. Thiel, P.R. Jones, Metab. Eng. Commun., 2 (2015) 15 .

10. M. Sakuth, T. Mensing, J. Schuler, W. Heitmann, G. Strehlke, D. Mayer, Ethers aliphatics, (2011) Ullmann's Encyclopedia of Industrial Chemistry, Weinheim: Wiley$\mathrm{VCH}, 7^{\text {th }}$ ed., vol. 13 , p. 436

11. C. Casas, R. Bringué, E. Ramírez, M. Iborra, J. Tejero, Appl. Catal A, 396 (2011) 129-139.

12. C. Casas, J. Guilera, E. Ramírez, R. Bringué, M. Iborra, J. Tejero, Biomass. Conv. Bioref., 3 (2012) 27-37.

13. C. Casas, C. Fité, M. Iborra, J. Tejero, F. Cunill, J. Chem. Eng. Data, 58 (2013) 741748.

14. I. Hoek, T.A. Nijhuis, A.I. Stankiewicz, J.A. Moulijn, Appl. Catal. A, 266 (2004) 109-116.

15. G.A. Olah, T. Shamma, G.K.S. Prakash, Catal. Lett., 46 (1997) 1-4.

16. A.N. Parvulescu, P.J.C. Hausoul, P.C.A. Bruijnincx, R.J.M. Klein-Gebbink, B.M. Weckhuysen, Catal. Today, 158 (2010) 130-138.

17. R. Bringué, E. Ramírez, C. Fité, M. Iborra, J. Tejero, Ind. Eng. Chem. Res., 50 (2011) 7911-7919.

18. A. Guyot, D.C. Sherrington, in: P.Hodge (Ed.), Syntheses and Separations Using Polymers Supports, Wiley, Chichester, 1988, (chapter 1), p. 15.

19. K. Jerabek, Anal. Chem., 57 (1985) 1598-1602.

20. A.G. Ogston, Trans. Faraday Soc., 54 (1958) 1754-1757.

21. K. Jerabeck, Kem. Ind., 62 (2013) 171-176.

22. R. Bringué, E. Ramírez, M. Iborra, J. Tejero, F. Cunill, J. Catal., 304 (2013) 7-21. 
23. R. Bringué, M. Cadenas, C. Fité, M. Iborra, F. Cunill, Chem. Eng. J., 207-208 (2012) 226-234.

24. M. Marchionna, M. Di Girolamo, R. Patrini, Catal. Today, 65 (2001) 397-413. 


\section{FIGURE CAPTIONS}

Figure 1. Reaction scheme in the dehydration of 1-octanol to DNOE

Figure 2. Morphology changes of gel-type and macroporous resins on swelling ${ }^{21}$.

Figure 3. Morphology of the tested resins swollen in water as shown by ISEC for medium and high DVB\% macroreticular resins.

Figure 4. Morphology of the tested resins swollen in water as shown by ISEC for microporous and low DVB\% macroreticular resins.

Figure 5. Temperature profile of the reacting system versus removed water after $7 \mathrm{~h}$ on 9 g of catalyst for CT224 ( ) and A-36 ( ) and atmospheric pressure.

Figure 6. Evolution of $\mathrm{mL}$ of collected $\mathrm{H}_{2} \mathrm{O}(\boldsymbol{\Delta}, \bullet \leftarrow)$ and reactor temperature $(\boldsymbol{\Delta}, \bullet \rightarrow)$ on $9 \mathrm{~g}$ of catalyst and atmospheric pressure for resins A-36 ( $\bullet$ ) and CT224 ( $\Delta$ ).

Figure 7. Evolution of boiling temperature versus $\mathrm{mL}$ of collected $\mathrm{H}_{2} \mathrm{O}$ for all tested resins on $9 \mathrm{~g}$ of resin and atmopheric pressure

Figure 8. Evolution of volume of water collected for the different resins over time on $9 \mathrm{~g}$ of resin and atmopheric pressure

Figure 9. Selectivity to DNOE $(\diamond), \mathrm{C}_{8}$ olefins $(\boldsymbol{\nabla})$ and C16 dimers $(\boldsymbol{\Delta})$ with respect to 1octanol vs. $\left[\mathrm{H}^{+}\right] / \mathrm{V}_{\text {sp }}$ ratio at $\mathrm{t}=7 \mathrm{~h}$ and on $9 \mathrm{~g}$ of catalyst for all the resins that reach complete 1-octanol conversion and atmopheric pressure. 


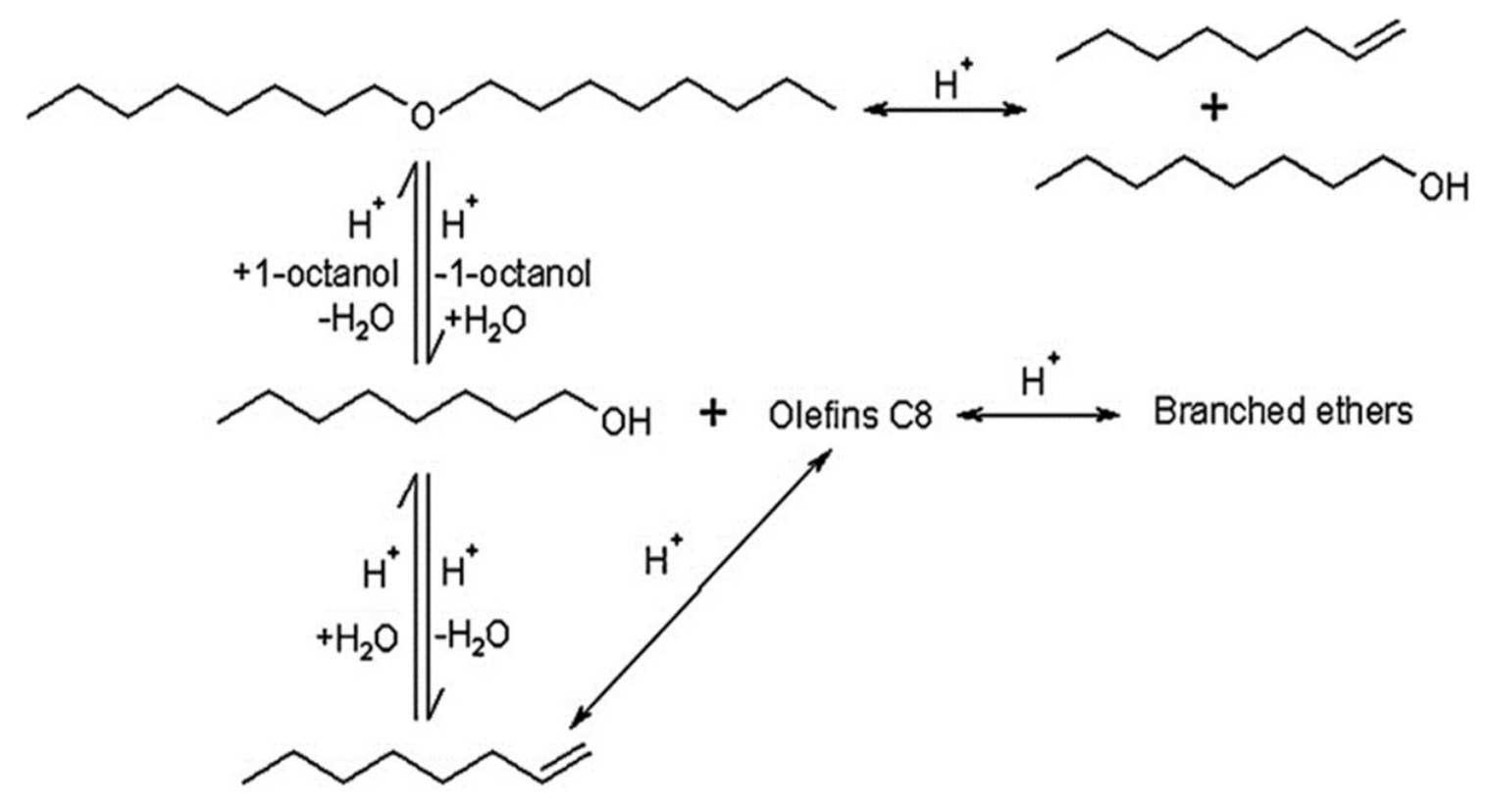

Figure 1. Reaction scheme in the dehydration of 1-octanol to DNOE 


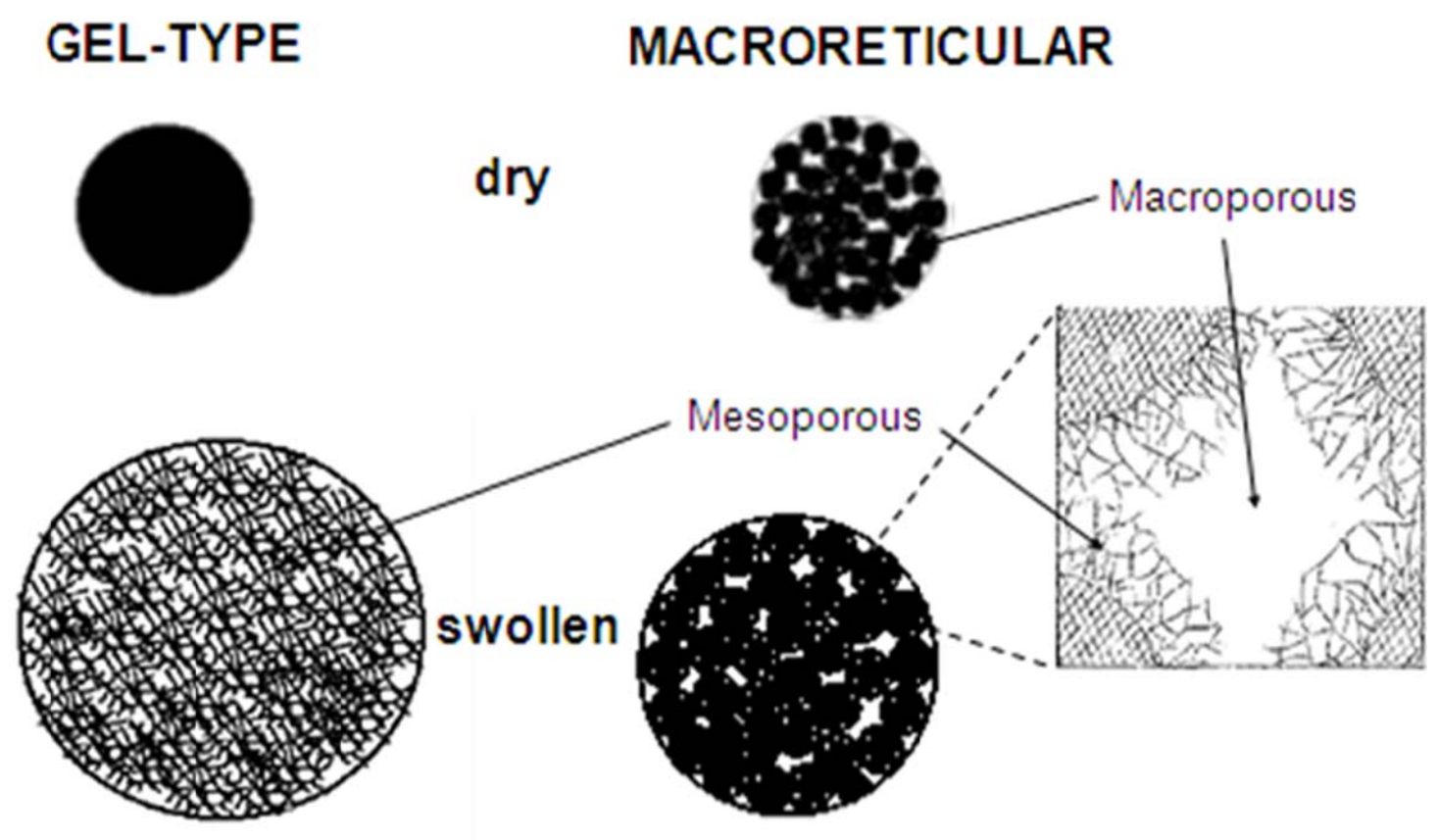

Figure 2. Morphology changes of gel-type and macroporous resins on swelling ${ }^{21}$ 


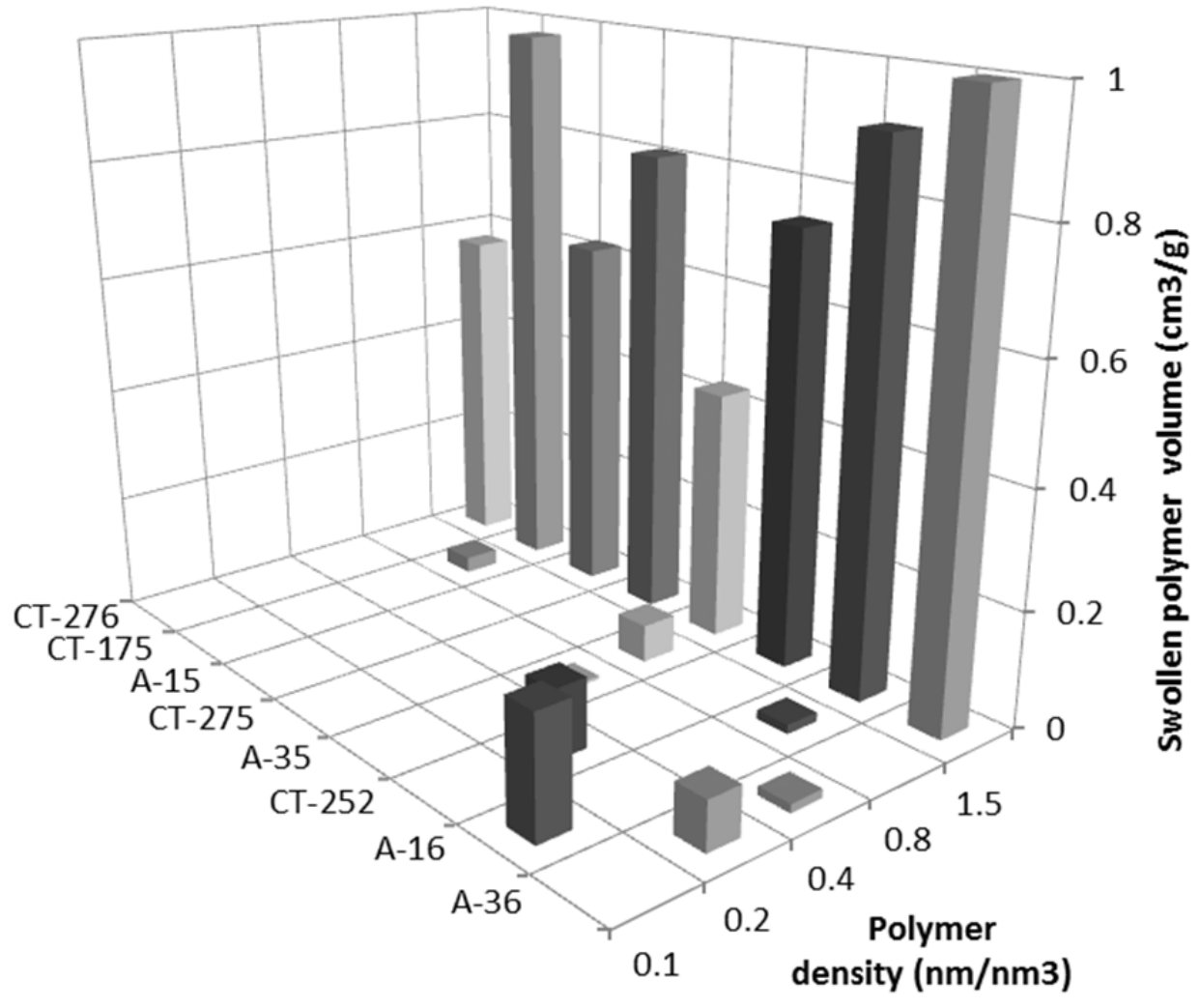

Figure 3. Morphology of the tested resins swollen in water as shown by ISEC for medium and high DVB\% macroreticular resins. 


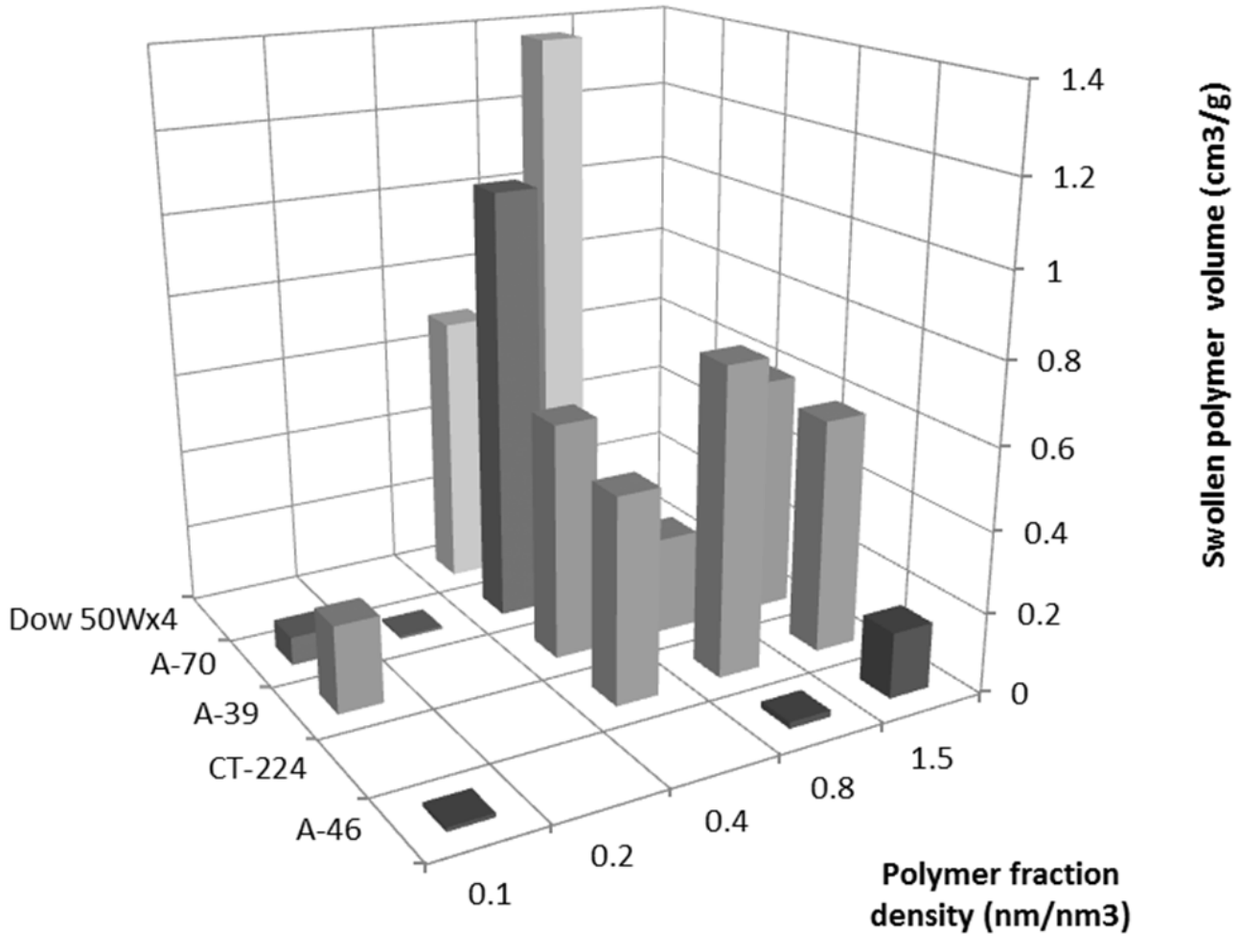

Figure 4. Morphology of the tested resins swollen in water as shown by ISEC for microporous and low DVB\% macroreticular resins. 


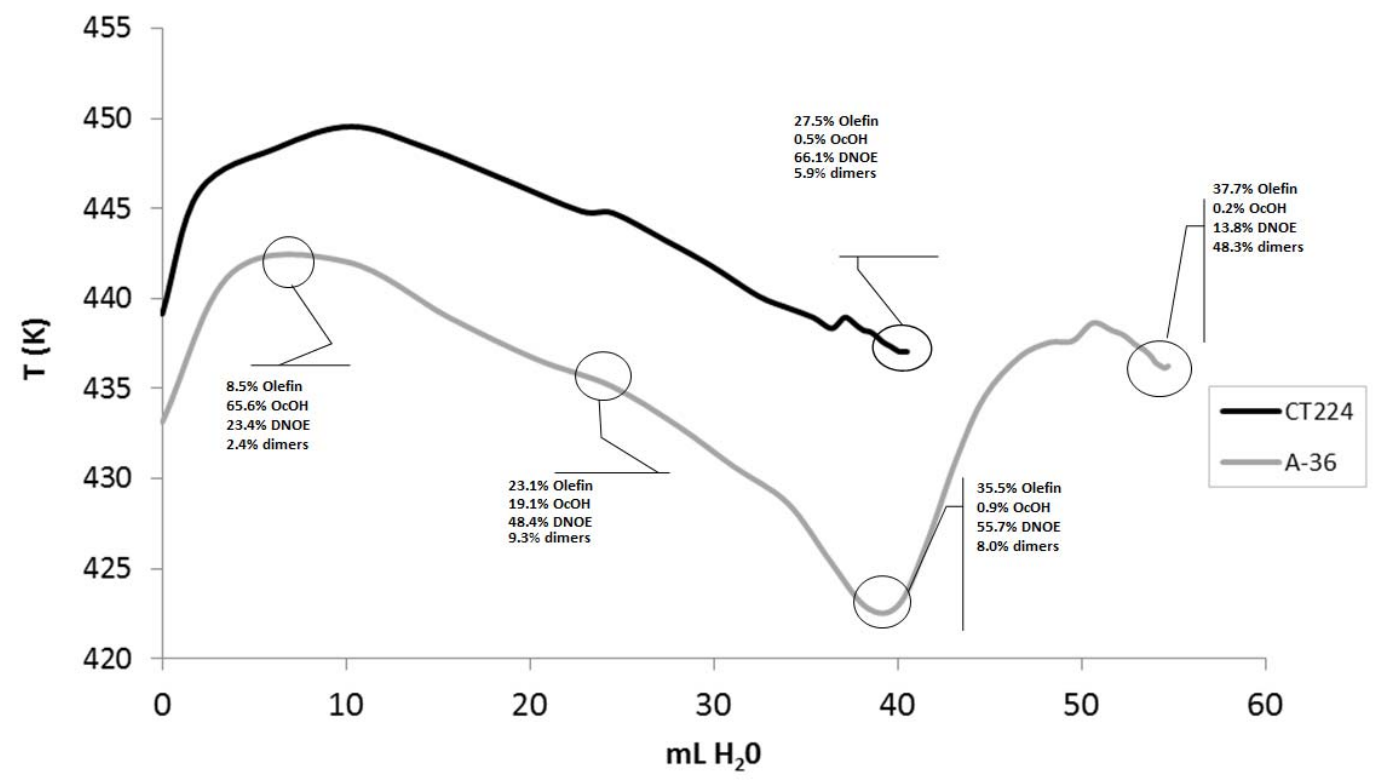

Figure 5. Temperature profile of the reacting system versus removed water after $7 \mathrm{~h}$ on 9 g of catalyst for CT224 ( T) and A-36 ( ) and atmospheric pressure. 


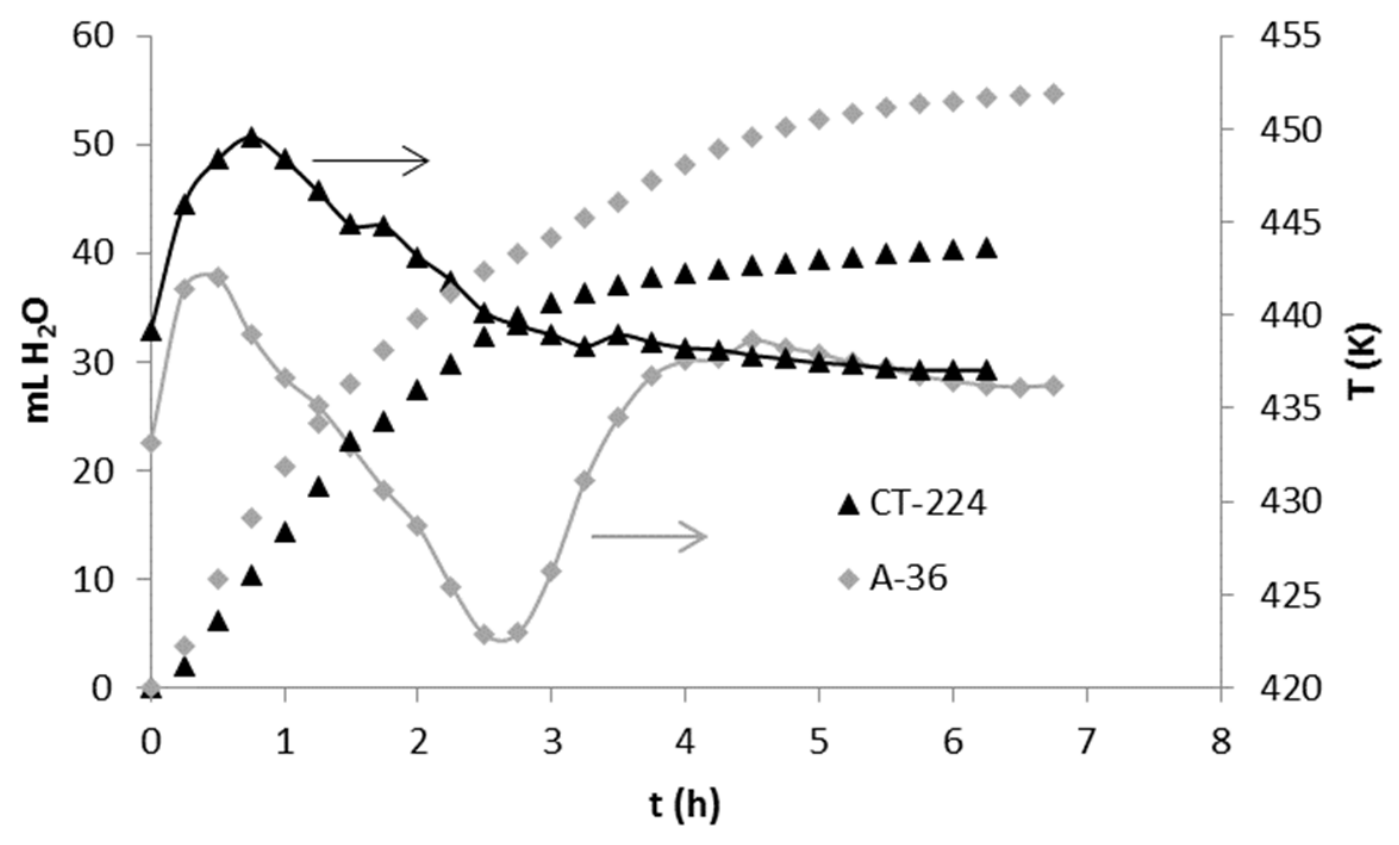

Figure 6. Evolution of $\mathrm{mL}$ of collected $\mathrm{H}_{2} \mathrm{O}(\boldsymbol{\Delta}, \downarrow \leftarrow)$ and reactor temperature $(\boldsymbol{\Delta}, \bullet \rightarrow)$ on $9 \mathrm{~g}$ of catalyst and atmospheric pressure for resins A-36 ( $\bullet)$ and CT224 (A). 


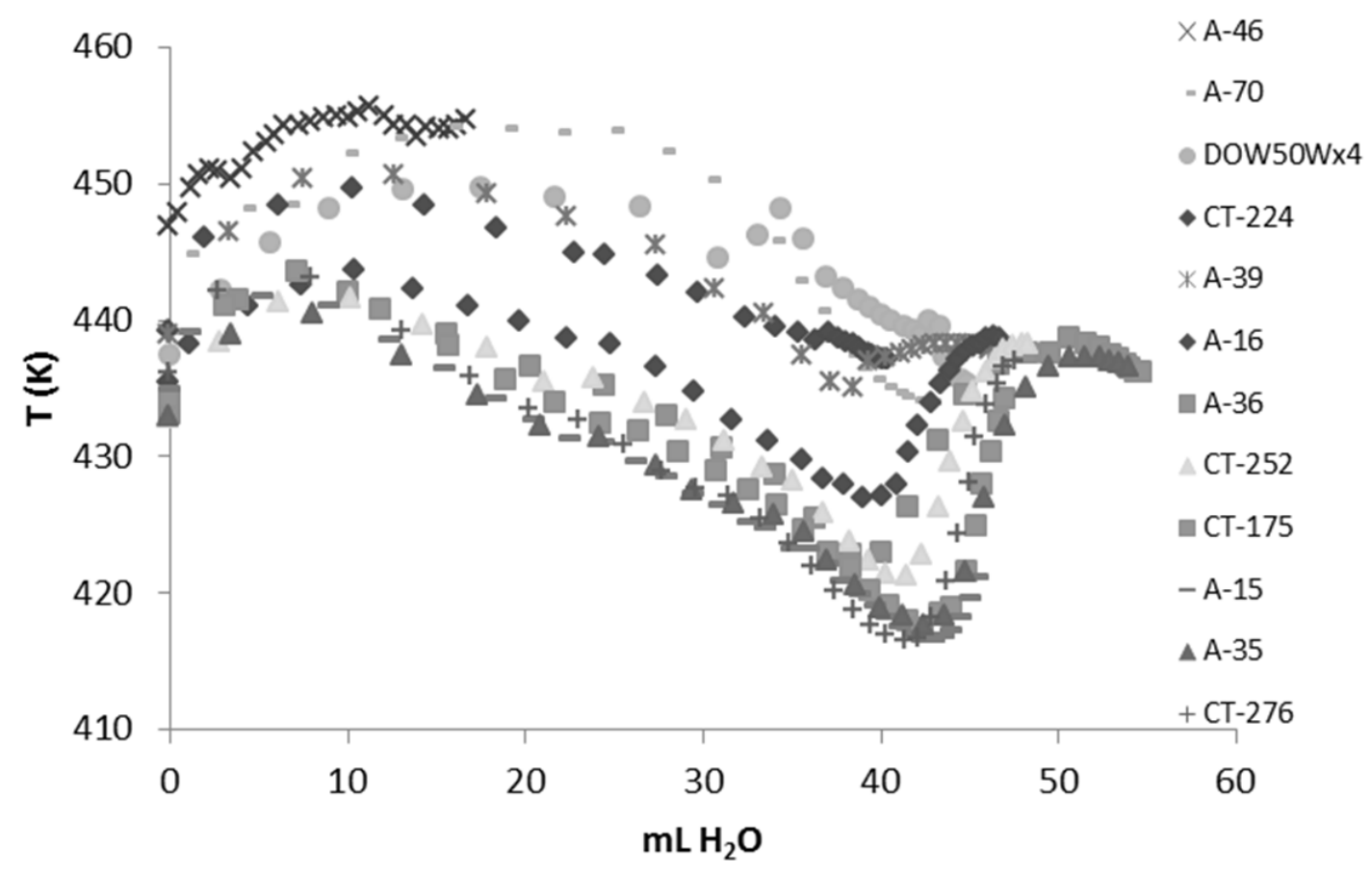

Figure 7. Evolution of boiling temperature versus $\mathrm{mL}$ of collected $\mathrm{H}_{2} \mathrm{O}$ for all tested resins on $9 \mathrm{~g}$ of resin and atmopheric pressure 


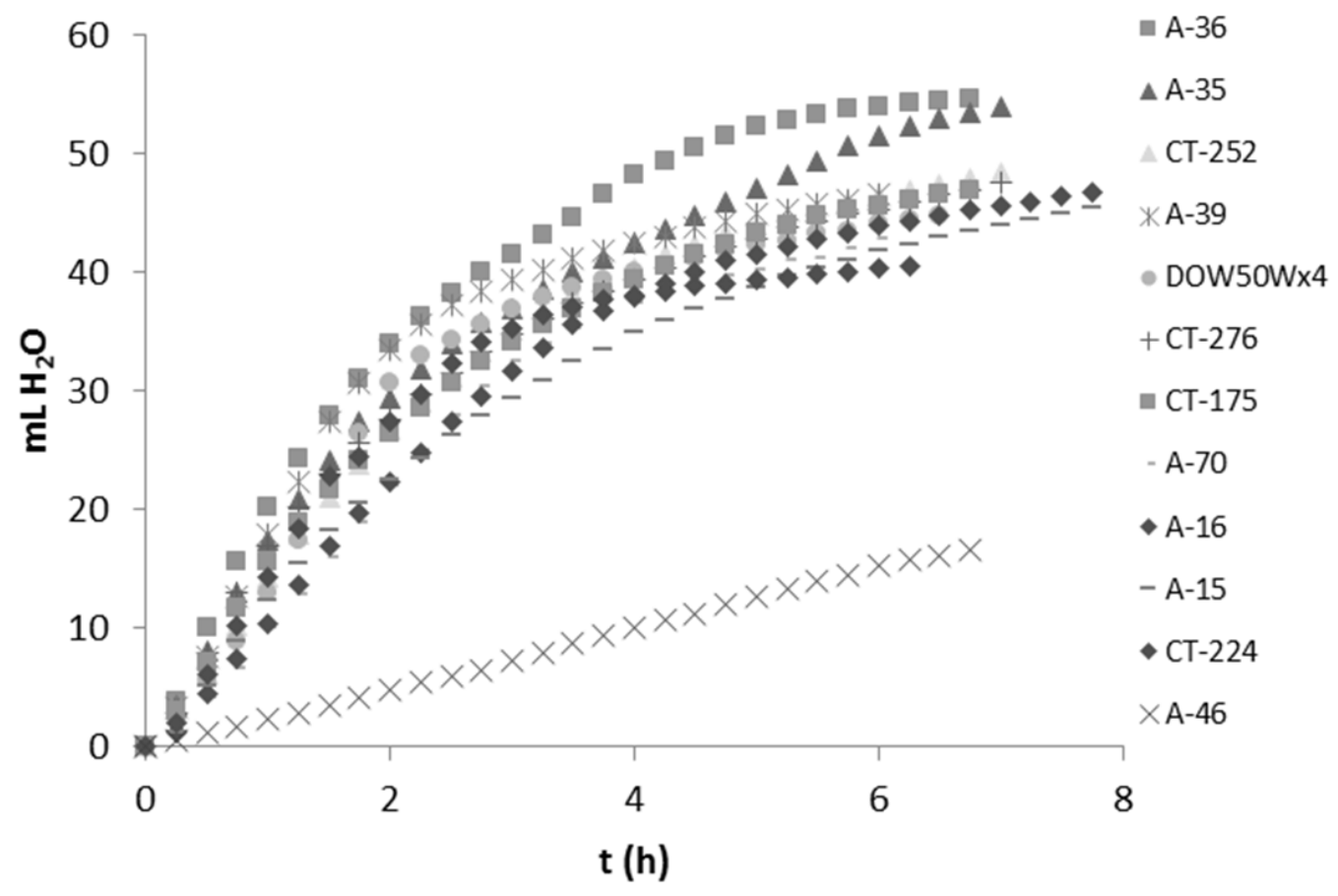

Figure 8. Evolution of volume of water collected for the different resins over time on $9 \mathrm{~g}$ of resin and atmopheric pressure 


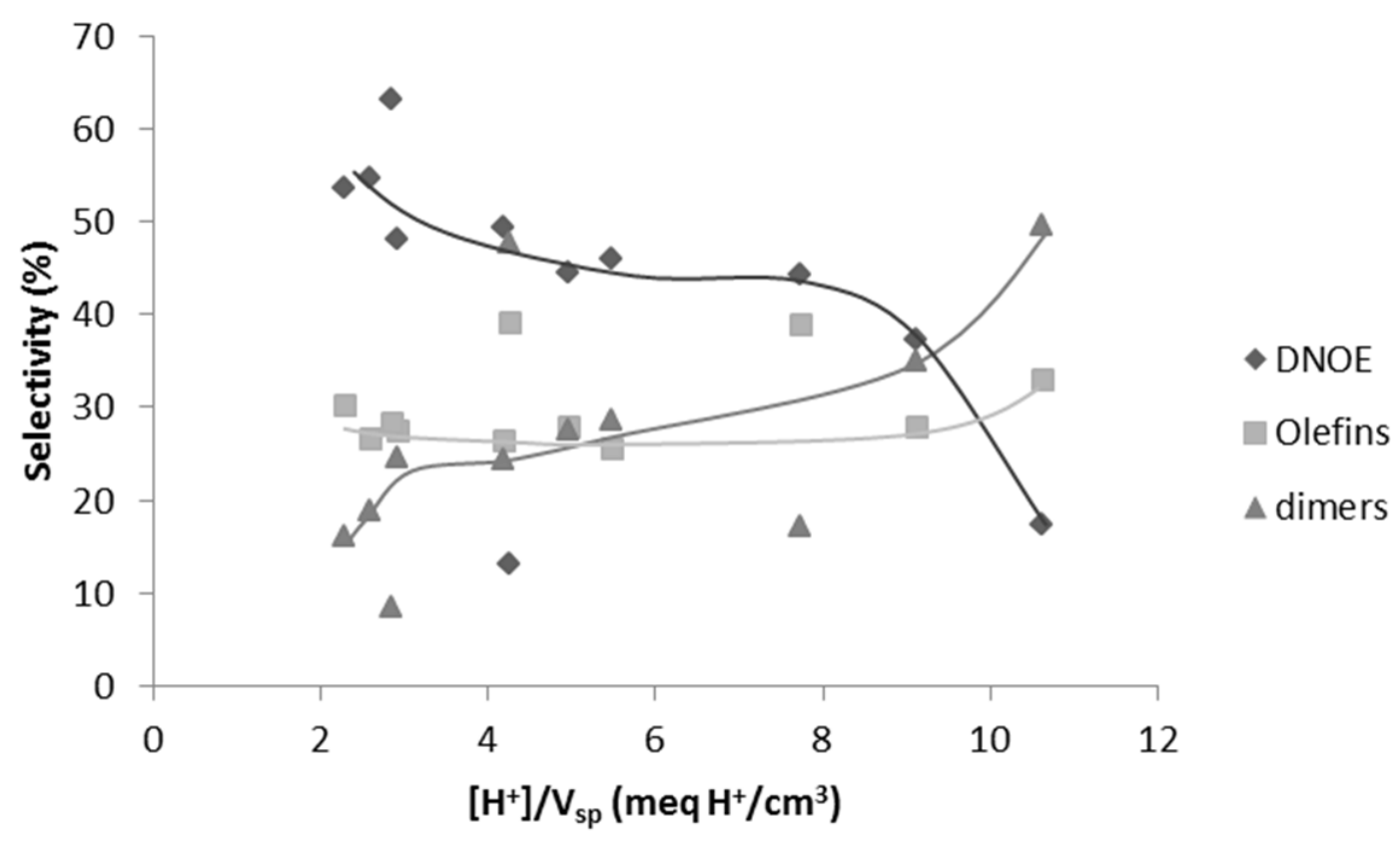

Figure 9. Selectivity to DNOE ( $\bullet$ ), C8 olefins ( $\bullet$ ) and C16 dimers ( $\Delta$ ) with respect to 1octanol vs. $\left[\mathrm{H}^{+}\right] / \mathrm{V}_{\text {sp }}$ ratio at $\mathrm{t}=7 \mathrm{~h}$ and on $9 \mathrm{~g}$ of catalyst for all the resins that reach complete 1-octanol conversion and atmopheric pressure. 